Copyright (C) 2014 IEEE. Personal use of this material is permitted. Permission from IEEE must be obtained for all other uses, in any current or future media, including reprinting/republishing this material for advertising or promotional purposes, creating new collective works, for resale or redistribution to servers or lists, or reuse of any copyrighted component of this work in other works. 


\title{
On Uplink-Downlink Sum-MSE Duality of Multi-hop MIMO Relay Channel
}

\author{
Ali Cagatay Cirik*, Muhammad R. A. Khandaker ${ }^{\dagger}$, Yue Rong $^{\ddagger}$ and Yingbo Hua* \\ * Department of Electrical Engineering, University of California Riverside, Riverside, CA, 92521 \\ $\dagger$ Department of Electronic \& Electrical Engineering, University College London, London, WC1E 7JE, UK \\ $\ddagger$ Department of Electrical and Computer Engineering, Curtin University, Bentley, WA 6102, Australia \\ Email: \{acirik,yhua\}@ee.ucr.edu,m.khandaker@ucl.ac.uk,y.rong@curtin.edu.au
}

\begin{abstract}
In this paper, the uplink and downlink sum meansquared error (MSE) duality for multi-hop amplify-and-forward (AF) multiple-input multiple-output (MIMO) relay channels is established, which is a generalization of several sum-MSE duality results. Unlike the previous results that prove the duality by calculating the MSEs for each stream directly, we introduce an interesting perspective to the relation of the uplink-downlink duality based on the Karush-Kuhn-Tucker (KKT) conditions associated with both uplink and downlink transceiver design optimization problems.
\end{abstract}

Index Terms-Amplify-and-forward, uplink-downlink duality, MIMO relay.

\section{INTRODUCTION}

One of the key techniques to solve the downlink optimization problems is to transform the downlink problem into an uplink problem via uplink-downlink duality relationship, and solve it in the uplink domain since the uplink channel has a simpler mathematical structure, and less coupling of variables.

The MSE duality for a single-hop was established under a sum-power constraint when perfect channel state information (CSI) is available at all the nodes in the system in [1]-[2], and for imperfect CSI in [3]-[5]. It has been shown that any MSE point achievable in the uplink can also be achieved in the downlink under the sum-power constraint. Recently, the uplink-downlink sum-MSE duality for single-hop systems [1][5] has been extended to two-hop and multi-hop AF MIMO relay systems in [6] and [7], respectively. ${ }^{1}$

Due to the multi-hop topology, MSE is a complicated function of the source, relay and receiver matrices, which makes both the proof of duality and the optimization problems associated with multi-hop MIMO relay networks much more challenging than the existing works with simpler network topology. As a direct application of the duality results in [7], [8], the complicated downlink MIMO multi-hop transceiver (source precoding, relay amplifying and receiver matrices) design problems can be carried out efficiently by focusing on an equivalent uplink MIMO multi-hop relay system.

\section{A. Contributions of This Work}

1) MSE duality in [1]-[4] and [7] is established by calculating the MSE of each stream of all users directly. Here,

\footnotetext{
${ }^{1}$ Note that signal-to-interference-noise ratio (SINR) duality for multi-hop AF MIMO relay systems has been established in [8].
}

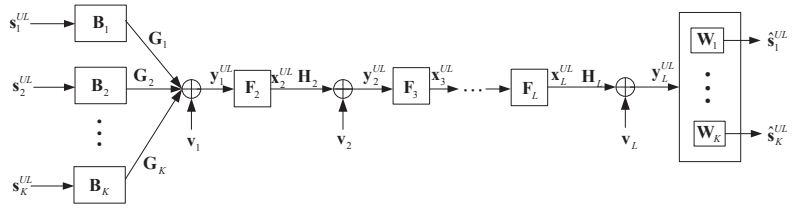

Fig. 1. Uplink multi-hop AF MIMO relay system.

we establish the uplink-downlink duality based on the KKT conditions of the uplink and downlink transceiver optimization problems, which is an interesting perspective to the relation of the uplink-downlink duality.

2) The duality result established in this paper generalizes the results in [5] and [6], which also use KKT conditions to prove the sum-MSE duality for single-hop and twohop MIMO channels, respectively.

3) The sum-MSE duality for multi-hop AF MIMO relay systems in [7] is established under the assumption that receivers employ linear minimum MSE (MMSE) receivers, the sum-MSE duality result in this paper is applicable to any kind of linear receiver.

The notations used in this paper are as follows. $(\cdot)^{T}$ and $(\cdot)^{H}$ denote transpose and conjugate transpose, respectively. $\mathbb{E}[\cdot], \mathbf{I}_{N}$ and $\operatorname{tr}(\cdot)$ denote the statistical expectation, $N \times N$ identity matrix and trace, respectively. For matrices $\mathbf{A}_{i}, \bigotimes_{i=l}^{k}\left(\mathbf{A}_{i}\right) \triangleq \mathbf{A}_{l} \ldots \mathbf{A}_{k}$. For example, $\bigotimes_{i=1}^{3}\left(\mathbf{A}_{i}\right) \triangleq$ $\mathbf{A}_{1} \mathbf{A}_{2} \mathbf{A}_{3}$ and $\bigotimes_{i=3}^{1}\left(\mathbf{A}_{i}\right) \triangleq \mathbf{A}_{3} \mathbf{A}_{2} \mathbf{A}_{1} . \prod_{i=l}^{k}\left(\mathbf{A}_{i}\right) \triangleq$ $\mathbf{A}_{l} \ldots \mathbf{A}_{k}$ for $l \leq k$ and is equal to identity matrix for $l>k$.

\section{SySTEM MOdEL}

Similar to the system model in [7]-[8], we consider a wireless communication system with $K$ users, $L-1(L \geq 2)$ half-duplex AF relay nodes, and one base station (BS) node, where each node is equipped with multiple antennas. The number of antennas at the $l$ th relay node of the uplink system is $N_{l}, l=1, \ldots, L-1$ and the BS is equipped with $N_{L}$ antennas. Due to the path-loss in the wireless channels, we assume that the signal transmitted by the $l$ th node can only be received by the $(l+1)$ th node, so the signal transmitted from the source node travel through $L$ hops to reach to its destination. The $i$ th user is equipped with $M_{i}$ antennas, and transmits (receives) $M_{i}$ independent data streams. 


\section{A. Uplink MIMO Relay System}

The uplink MIMO multi-hop relay system is shown in Fig. 1. The data streams $\mathbf{s}_{i}^{U L} \in C^{M_{i} \times 1}$ is linearly precoded by the $i$ th user with the source precoding matrix $\mathbf{B}_{i} \in C^{M_{i} \times M_{i}}$ and the $i$ th user transmits the precoded signal vector $\mathbf{u}_{i}=$ $\mathbf{B}_{i} \mathbf{s}_{i}^{U L}$ to the first relay node. We assume complex, zero mean, independent and identically distributed (i.i.d.) data streams with $\mathbb{E}\left[\mathbf{s}_{i}^{U L}\left(\mathbf{s}_{i}^{U L}\right)^{H}\right]=\mathbf{I}_{M_{i}}$. The received signal at the first relay node is given by

$$
\mathbf{y}_{1}^{U L}=\sum_{i=1}^{K} \mathbf{G}_{i} \mathbf{B}_{i} \mathbf{s}_{i}^{U L}+\mathbf{v}_{1}
$$

where $\mathbf{G}_{i} \in C^{N_{1} \times M_{i}}, i=1, \ldots, K$, is the channel between the first relay node and the $i$ th user and $\mathbf{v}_{1}$ is the $N_{1} \times 1$ i.i.d. additive white Gaussian noise (AWGN) vector at the first relay.

The $l$ th relay node, $l=1, \ldots, L-1$, applies $\mathbf{F}_{l+1} \in$ $C^{N_{l} \times N_{l}}$ to amplify and forward the received signals, which is given by

$$
\mathbf{x}_{l+1}^{U L}=\mathbf{F}_{l+1} \mathbf{y}_{l}^{U L}, \quad l=1, \ldots, L-1
$$

where $\mathbf{y}_{l}^{U L} \in C^{N_{l} \times 1}$ is the signal that $l$ th relay node receives, $l=1, \ldots, L-1$. From (1) and (2), the received signal vector at the relay nodes, $l=1, \ldots, L-1$, and the received signal vector at the BS $(l=L)$ can be written as

$$
\mathbf{y}_{l}^{U L}=\mathbf{A}_{l} \sum_{i=1}^{K} \mathbf{G}_{i} \mathbf{B}_{i} \mathbf{s}_{i}^{U L}+\overline{\mathbf{v}}_{l}, \quad l=1, \ldots, L
$$

where $\mathbf{A}_{l}$ is the equivalent channel matrix between the first relay node and the $l$ th relay node, and $\overline{\mathbf{v}}_{l}$ is the equivalent noise vector given by

$$
\begin{aligned}
\mathbf{A}_{l} & = \begin{cases}\bigotimes_{i=l}^{2}\left(\mathbf{H}_{i} \mathbf{F}_{i}\right), & l=2, \ldots, L \\
\mathbf{I}_{N_{1}}, & l=1,\end{cases} \\
\overline{\mathbf{v}}_{l} & = \begin{cases}\sum_{j=2}^{l}\left(\bigotimes_{i=l}^{j}\left(\mathbf{H}_{i} \mathbf{F}_{i}\right) \mathbf{v}_{j-1}\right)+\mathbf{v}_{l}, & l=2, \ldots, L \\
\mathbf{v}_{1}, & l=1 .\end{cases}
\end{aligned}
$$

Here $\mathbf{H}_{l} \in C^{N_{l} \times N_{l-1}}, l=2, \ldots, L$, is the channel matrix at the $l$ th hop, and $\mathbf{v}_{l}$ is the i.i.d. AWGN at the $(l+1)$-th node of the uplink system, $l=1, \ldots, L$. We assume that all noises are complex signals with zero mean and unit variance.

From (5), the covariance matrix of $\overline{\mathbf{v}}_{l}$ can be written as,

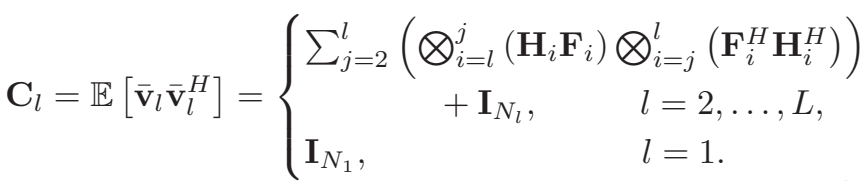

To estimate the data streams transmitted, the BS applies a linear receiver, i.e., $\hat{\mathbf{s}}_{j}^{U L}=\mathbf{W}_{j} \mathbf{y}_{L}^{U L}$, which is given by

$$
\hat{\mathbf{s}}_{j}^{U L}=\mathbf{W}_{j}\left[\mathbf{A}_{L} \sum_{i=1}^{K} \mathbf{G}_{i} \mathbf{B}_{i} \mathbf{s}_{i}^{U L}+\overline{\mathbf{v}}_{L}\right], \quad j=1, \ldots, K
$$

where $\mathbf{W}_{j}$ is the weight matrix of the linear receiver of size $M_{j} \times N_{L}$. From (3) and (7), the MSE matrix of the $j$ th user,

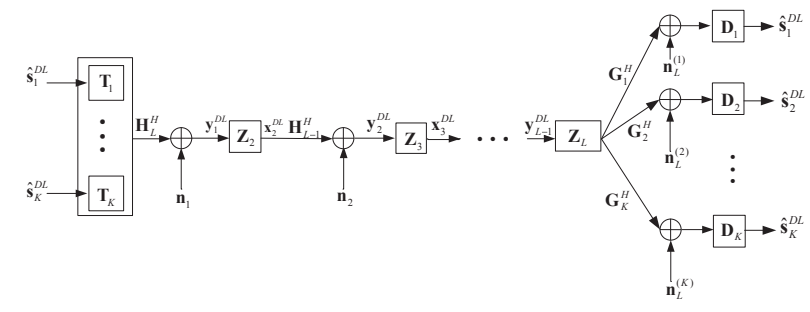

Fig. 2. Downlink multi-hop AF MIMO relay system.

$\mathbf{E}_{j}^{U L}=\mathbb{E}\left[\left(\mathbf{s}_{j}^{U L}-\hat{\mathbf{s}}_{j}^{U L}\right)\left(\mathbf{s}_{j}^{U L}-\hat{\mathbf{s}}_{j}^{U L}\right)^{H}\right], j=1, \ldots, K$ can be written as

$$
\begin{aligned}
\mathbf{E}_{j}^{U L}= & \mathbf{I}_{M_{j}}-\mathbf{W}_{j} \mathbf{A}_{L} \mathbf{G}_{j} \mathbf{B}_{j}-\mathbf{B}_{j}^{H} \mathbf{G}_{j}^{H} \mathbf{A}_{L}^{H} \mathbf{W}_{j}^{H} \\
& +\mathbf{W}_{j}\left[\mathbf{A}_{L} \mathbf{A}^{U L} \mathbf{A}_{L}^{H}+\mathbf{C}_{L}\right] \mathbf{W}_{j}^{H}
\end{aligned}
$$

where $\mathbf{A}^{U L}=\sum_{i=1}^{K} \mathbf{G}_{i} \mathbf{B}_{i} \mathbf{B}_{i}^{H} \mathbf{G}_{i}^{H}$.

The transmission power consumed at the $l$ th relay node is

$$
\operatorname{tr}\left(\mathbb{E}\left[\mathbf{x}_{l+1}^{U L}\left(\mathbf{x}_{l+1}^{U L}\right)^{H}\right]\right)=\operatorname{tr}\left(\mathbf{F}_{l+1}\left(\mathbf{A}_{l} \mathbf{A}^{U L} \mathbf{A}_{l}^{H}+\mathbf{C}_{l}\right) \mathbf{F}_{l+1}^{H}\right)(9)
$$

The uplink transceiver optimization problem is formulated as:

$$
\begin{array}{ll}
\min _{\mathbf{F}_{l}, \mathbf{B}_{j}, \mathbf{W}_{j}} \sum_{j=1}^{K} \operatorname{tr}\left(\mathbf{E}_{j}^{U L}\right) \\
\text { s.t. } \quad \sum_{j=1}^{K} \operatorname{tr}\left(\mathbf{B}_{j} \mathbf{B}_{j}^{H}\right) \leq P_{1}^{U L}, \\
\quad \operatorname{tr}\left(\mathbf{F}_{l}\left(\mathbf{A}_{l-1} \mathbf{A}^{U L} \mathbf{A}_{l-1}^{H}+\mathbf{C}_{l-1}\right) \mathbf{F}_{l}^{H}\right) \leq P_{l}^{U L}, \forall l
\end{array}
$$

where (11) and (12) are the total transmit power at the users and transmission power constraints at each relay node, respectively, and $P_{l}^{U L}, l=1, \ldots, L$, are the power limit.

\section{B. Downlink MIMO Relay System}

The downlink communication system is shown in Fig. 2. The BS linearly precodes the data streams of user $i, \mathbf{s}_{i}^{D L} \in$ $C^{M_{i} \times 1}$ with the matrix $\mathbf{T}_{i} \in C^{N_{L} \times M_{i}}$ and transmits the $N_{L} \times 1$ precoded signal vector $\sum_{i=1}^{K} \mathbf{T}_{i} \mathbf{s}_{i}^{D L}$. We assume complex data streams with zero mean, i.i.d data streams with $\mathbb{E}\left[\mathbf{s}_{i}^{D L}\left(\mathbf{s}_{i}^{D L}\right)^{H}\right]=\mathbf{I}_{M_{i}}$. The signal vector received of size $N_{L-1} \times 1$ at the first relay node of the downlink system can be written as

$$
\mathbf{y}_{1}^{D L}=\mathbf{H}_{L}^{H} \sum_{i=1}^{K} \mathbf{T}_{i} \mathbf{s}_{i}^{D L}+\mathbf{n}_{1}
$$

where $\mathbf{n}_{1} \in C^{N_{L-1} \times 1}$ is the AWGN vector at the first relay.

The $l$ th relay node in the downlink system, $l=1, \ldots, L-$ 1 applies $\mathbf{Z}_{l+1} \in C^{N_{L-l} \times N_{L-l}}$ to amplify and forward the received signals, i.e., $\mathbf{x}_{l+1}^{D L}=\mathbf{Z}_{l+1} \mathbf{y}_{l}^{D L}, l=1, \ldots, L-1$, where $\mathbf{y}_{l}^{D L} \in C^{N_{L-l} \times 1}, l=1, \ldots, L-1$, is the received signal vector at the $l$ th relay node in the downlink channel and is expressed as

$$
\mathbf{y}_{l}^{D L}=\mathbf{K}_{l} \mathbf{H}_{L}^{H} \sum_{i=1}^{K} \mathbf{T}_{i} \mathbf{s}_{i}^{D L}+\overline{\mathbf{n}}_{l}, \quad l=1, \ldots, L-1 .(14)
$$


Here $\mathbf{K}_{l}$ is the equivalent channel matrix between the first relay node and the $l$ th relay node in the downlink channel and $\overline{\mathbf{n}}_{l}$ is the equivalent noise vector given by

$$
\begin{aligned}
& \mathbf{K}_{l}= \begin{cases}\bigotimes_{m=L-l+1}^{L-1}\left(\mathbf{H}_{m}^{H} \mathbf{Z}_{L-m+1}\right), & l=2, \ldots, L-1 \\
\mathbf{I}_{N_{L-1},} & l=1,\end{cases} \\
& \overline{\mathbf{n}}_{l}=\left\{\begin{array}{cc}
\sum_{k=1}^{l-1} \bigotimes_{m=L-l+1}^{L-k}\left(\mathbf{H}_{m}^{H} \mathbf{Z}_{L-m+1}\right) \mathbf{n}_{k} \\
+\mathbf{n}_{l}, & l=2, \ldots, L-1 \\
\mathbf{n}_{1}, & l=1
\end{array}\right.
\end{aligned}
$$

where $\mathbf{n}_{l}$ is the i.i.d. AWGN vector at the $l$ th relay node, $l=1, \ldots, L-1$. The received signal vector at the $i$ th user $i=1, \ldots, K$ can be expressed as

$$
\begin{aligned}
\mathbf{y}_{L}^{(i)} & =\mathbf{G}_{i}^{H} \mathbf{Z}_{L} \mathbf{y}_{L-1}^{D L}+\mathbf{n}_{L}^{(i)} \\
& =\mathbf{G}_{i}^{H} \mathbf{Z}_{L} \mathbf{K}_{L-1} \mathbf{H}_{L}^{H} \sum_{i=1}^{K} \mathbf{T}_{i} \mathbf{s}_{i}^{D L}+\overline{\mathbf{n}}_{L}^{(i)}
\end{aligned}
$$

where $\overline{\mathbf{n}}_{L}^{(i)}=\mathbf{G}_{i}^{H} \mathbf{Z}_{L} \overline{\mathbf{n}}_{L-1}+\mathbf{n}_{L}^{(i)}$ is the equivalent noise vector at the $i$ th user.

From (16), the covariance matrix of $\overline{\mathbf{n}}_{l}, \mathbf{C}_{l}^{D L}$, at the $l$ th relay node, $l=2, \ldots, L-1$ and the covariance matrix of $\overline{\mathbf{n}}_{L}^{(i)}, \mathbf{C}_{L}^{(i)}$, at the $i$ th user can be written as

$$
\begin{aligned}
& \mathbf{C}_{l}^{D L}=\sum_{k=1}^{l-1}\left(\bigotimes_{m=L-l+1}^{L-k}\left(\mathbf{H}_{m}^{H} \mathbf{Z}_{L-m+1}\right) \bigotimes_{m=L-k}^{L-l+1}\left(\mathbf{Z}_{L-m+1}^{H} \mathbf{H}_{m}\right)\right) \\
& +\mathbf{I}_{N_{L-l}}, \quad l=2, \ldots, L-1, \\
& \mathbf{C}_{L}^{(i)}=\mathbf{G}_{i}^{H} \mathbf{Z}_{L} \mathbf{C}_{L-1}^{D L} \mathbf{Z}_{L}^{H} \mathbf{G}_{i}+\mathbf{I}_{M_{i}} .
\end{aligned}
$$

To estimate the data streams $\mathbf{s}_{j}^{D L}, j$ th user applies a linear receiver matrix $\mathbf{D}_{j} \in C^{M_{j} \times M_{j}}$, i.e., $\hat{\mathbf{s}}_{j}^{D L}=\mathbf{D}_{j} \mathbf{y}_{L}^{(j)}, j=$ $1, \ldots, K$, which is written as

$$
\hat{\mathbf{s}}_{j}^{D L}=\mathbf{D}_{j} \mathbf{G}_{j}^{H} \mathbf{Z}_{L} \mathbf{K}_{L-1} \mathbf{H}_{L}^{H} \sum_{i=1}^{K} \mathbf{T}_{i} \mathbf{s}_{i}^{D L}+\mathbf{D}_{j} \overline{\mathbf{n}}_{L}^{(j)} .
$$

The MSE matrix of the $j$ th user, $j=1, \ldots, K$, i.e., $\mathbf{E}_{j}^{D L}=$ $\mathbb{E}\left[\left(\mathbf{s}_{j}^{D L}-\hat{\mathbf{s}}_{j}^{D L}\right)\left(\mathbf{s}_{j}^{D L}-\hat{\mathbf{s}}_{j}^{D L}\right)^{H}\right]$ can be written as

$$
\begin{aligned}
& \mathbf{E}_{j}^{D L} \\
& =\mathbf{I}_{M_{j}}-\mathbf{D}_{j} \mathbf{G}_{j}^{H} \mathbf{Z}_{L} \mathbf{K}_{L-1} \mathbf{H}_{L}^{H} \mathbf{T}_{j}-\mathbf{T}_{j}^{H} \mathbf{H}_{L} \mathbf{K}_{L-1}^{H} \mathbf{Z}_{L}^{H} \mathbf{G}_{j} \mathbf{D}_{j}^{H} \\
& +\mathbf{D}_{j}\left[\mathbf{G}_{j}^{H} \mathbf{Z}_{L} \mathbf{K}_{L-1} \mathbf{A}^{D L} \mathbf{K}_{L-1}^{H} \mathbf{Z}_{L}^{H} \mathbf{G}_{j}+\mathbf{C}_{L}^{(j)}\right] \mathbf{D}_{j}^{H}
\end{aligned}
$$

where $\mathbf{A}^{D L}=\mathbf{H}_{L}^{H} \sum_{i=1}^{K} \mathbf{T}_{i} \mathbf{T}_{i}^{H} \mathbf{H}_{L}$.

The transmission power consumed at the $l$ th relay node

$\operatorname{tr}\left(\mathbb{E}\left[\mathbf{x}_{l+1}^{D L}\left(\mathbf{x}_{l+1}^{D L}\right)^{H}\right]\right)=\operatorname{tr}\left(\mathbf{Z}_{l+1}\left(\mathbf{K}_{l} \mathbf{A}^{D L} \mathbf{K}_{l}^{H}+\mathbf{C}_{l}^{D L}\right) \mathbf{Z}_{l+1}^{H}\right)$.

The downlink transceiver optimization problem is formulated:

$$
\begin{aligned}
\min _{\mathbf{Z}_{l}, \mathbf{T}_{j}, \mathbf{D}_{j}} & \sum_{j=1}^{K} \operatorname{tr}\left(\mathbf{E}_{j}^{D L}\right) \\
\text { s.t. } & \sum_{j=1}^{K} \operatorname{tr}\left(\mathbf{T}_{j} \mathbf{T}_{j}^{H}\right) \leq P_{1}^{D L} \\
& \operatorname{tr}\left(\mathbf{Z}_{l}\left(\mathbf{K}_{l-1} \mathbf{A}^{D L} \mathbf{K}_{l-1}^{H}+\mathbf{C}_{l-1}^{D L}\right) \mathbf{Z}_{l}^{H}\right) \leq P_{l}^{D L}, \forall l
\end{aligned}
$$

where (23) and (24) are the total transmit power at the users and transmission power constraints at each relay node, respectively, and $P_{l}^{D L}, l=1, \ldots, L$, are the power limit.

\section{UPLINK-DOWNLINK DUALITY}

The optimization problems (10)-(12) and (22)-(24) are both non-convex, but the objective functions and constraints of them are continuously differentiable. Thus the uplink-downlink duality can be established based on their KKT conditions [5].

\section{A. The KKT Conditions of the Uplink Problem}

The Lagrangian function of (10)-(12) can be written as

$$
\begin{aligned}
\mathcal{L}^{U L} & =\sum_{j=1}^{K} \operatorname{tr}\left(\mathbf{E}_{j}^{U L}\right)+\lambda_{1}\left(\sum_{j=1}^{K} \operatorname{tr}\left(\mathbf{B}_{j} \mathbf{B}_{j}^{H}\right)-P_{1}^{U L}\right) \\
& +\sum_{l=2}^{L} \lambda_{l}\left(\operatorname{tr}\left(\mathbf{F}_{l}\left(\mathbf{A}_{l-1} \mathbf{A}^{U L} \mathbf{A}_{l-1}^{H}+\mathbf{C}_{l-1}\right) \mathbf{F}_{l}^{H}\right)-P_{l}^{U L}\right)
\end{aligned}
$$

where $\lambda_{1}$ and $\lambda_{l}, l=2, \ldots, L$, are the Lagrange multipliers of the power constraints in (11) and (12). The gradient function of (25) with respect to $\mathbf{B}_{k}, \mathbf{F}_{l}, \mathbf{W}_{k}$ is given by

$$
\begin{gathered}
\mathbf{G}_{k}^{H} \mathbf{A}_{L}^{H} \mathbf{W}_{k}^{H}=\left(\lambda_{1} \mathbf{I}_{M_{k}}+\sum_{l=2}^{L} \lambda_{l} \mathbf{G}_{k}^{H} \mathbf{A}_{l-1}^{H} \mathbf{F}_{l}^{H} \mathbf{F}_{l} \mathbf{A}_{l-1} \mathbf{G}_{k}\right. \\
\left.+\sum_{j=1}^{K} \mathbf{G}_{k}^{H} \mathbf{A}_{L}^{H} \mathbf{W}_{j}^{H} \mathbf{W}_{j} \mathbf{A}_{L} \mathbf{G}_{k}\right) \mathbf{B}_{k}
\end{gathered}
$$

$\sum_{j=1}^{K} \mathbf{H}_{k}^{H} \prod_{m=k+1}^{L}\left(\mathbf{F}_{m}^{H} \mathbf{H}_{m}^{H}\right) \mathbf{W}_{j}^{H} \mathbf{B}_{j}^{H} \mathbf{G}_{j}^{H} \prod_{m=2}^{k-1}\left(\mathbf{F}_{m}^{H} \mathbf{H}_{m}^{H}\right)=$

$\sum_{j=1}^{K} \mathbf{H}_{k}^{H} \prod_{m=k+1}^{L}\left(\mathbf{F}_{m}^{H} \mathbf{H}_{m}^{H}\right) \mathbf{W}_{j}^{H} \mathbf{W}_{j} \mathbf{A}_{L} \mathbf{A}^{U L} \prod_{m=2}^{k-1}\left(\mathbf{F}_{m}^{H} \mathbf{H}_{m}^{H}\right)$

$+\sum_{j=1}^{K} \sum_{m=2}^{k} \mathbf{H}_{k}^{H} \prod_{l=k+1}^{L}\left(\mathbf{F}_{l}^{H} \mathbf{H}_{l}^{H}\right) \mathbf{W}_{j}^{H} \mathbf{W}_{j} \bigotimes_{l=L}^{m}\left(\mathbf{H}_{l} \mathbf{F}_{l}\right)$

$\times \prod_{l=m}^{k-1}\left(\mathbf{F}_{l}^{H} \mathbf{H}_{l}^{H}\right)+\lambda_{k} \mathbf{F}_{k}\left(\mathbf{A}_{k-1} \mathbf{A}^{U L} \mathbf{A}_{k-1}^{H}+\mathbf{C}_{k-1}\right)$

$+\sum_{l=k+1}^{L} \lambda_{l}\left(\mathbf{H}_{k}^{H} \prod_{m=k+1}^{l-1}\left(\mathbf{F}_{m}^{H} \mathbf{H}_{m}^{H}\right) \mathbf{F}_{l}^{H} \mathbf{F}_{l} \mathbf{A}_{l-1} \mathbf{A}^{U L}\right.$

$\times \prod_{m=2}^{k-1}\left(\mathbf{F}_{m}^{H} \mathbf{H}_{m}^{H}\right)+\sum_{j=2}^{k} \mathbf{H}_{k}^{H} \prod_{m=k+1}^{l-1}\left(\mathbf{F}_{m}^{H} \mathbf{H}_{m}^{H}\right) \mathbf{F}_{l}^{H} \mathbf{F}_{l}$

$\left.\times \bigotimes_{i=l-1}^{j}\left(\mathbf{H}_{i} \mathbf{F}_{i}\right) \prod_{m=j}^{k-1}\left(\mathbf{F}_{m}^{H} \mathbf{H}_{m}^{H}\right)\right)$,

$$
\mathbf{B}_{k}^{H} \mathbf{G}_{k}^{H} \mathbf{A}_{L}^{H}=\mathbf{W}_{k}\left(\mathbf{A}_{L} \mathbf{A}^{U L} \mathbf{A}_{L}^{H}+\mathbf{C}_{L}\right)
$$

where we have used the identities from [9] that $\frac{\partial \operatorname{tr}\left(\mathbf{A} \mathbf{Z}^{H}\right)}{\partial \Re \mathbf{Z}}=$ $\mathbf{A}, \frac{\partial \operatorname{tr}(\mathbf{B Z})}{\partial \Re \mathbf{Z}}=\mathbf{B}^{T}, i \frac{\partial \operatorname{tr}\left(\mathbf{A} \mathbf{Z}^{H}\right)}{\partial \Im \mathbf{Z}}=\mathbf{A}, i \frac{\partial \operatorname{tr}(\mathbf{B Z})}{\partial \Im \mathbf{Z}}=-\mathbf{B}^{T}$ and 
$\frac{d f(z)}{d z^{*}}=\frac{1}{2}\left[\frac{\partial f(z)}{\partial \Re z}+i \frac{\partial f(z)}{\partial \Im z}\right]$. Here $i=\sqrt{-1}$. The other KKT conditions associated with (10)-(12) are given below

$$
\begin{aligned}
& \lambda_{1}\left(\sum_{j=1}^{K} \operatorname{tr}\left(\mathbf{B}_{j} \mathbf{B}_{j}^{H}\right)-P_{1}^{U L}\right)=0 \\
& \lambda_{l}\left(\operatorname{tr}\left(\mathbf{F}_{l}\left(\mathbf{A}_{l-1} \mathbf{A}^{U L} \mathbf{A}_{l-1}^{H}+\mathbf{C}_{l-1}\right) \mathbf{F}_{l}^{H}\right)-P_{l}^{U L}\right)=0 \\
& \lambda_{1} \geq 0, \quad \sum_{j=1}^{K} \operatorname{tr}\left(\mathbf{B}_{j} \mathbf{B}_{j}^{H}\right) \leq P_{1}^{U L} \\
& \lambda_{l} \geq 0, \quad \operatorname{tr}\left(\mathbf{F}_{l}\left(\mathbf{A}_{l-1} \mathbf{A}^{U L} \mathbf{A}_{l-1}^{H}+\mathbf{C}_{l-1}\right) \mathbf{F}_{l}^{H}\right) \leq P_{l}^{U L}
\end{aligned}
$$

Lemma 1. [Relation between the Lagrange multipliers, and the relay amplifying and receive matrices.]

For any solutions satisfying the KKT conditions (26)-(32), the Lagrange multipliers are

$$
\begin{aligned}
\lambda_{L} & =\frac{\sum_{k=1}^{K} \operatorname{tr}\left(\mathbf{W}_{k}^{H} \mathbf{W}_{k}\right)}{P_{L}^{U L}} \\
\lambda_{L-1} & =\frac{\operatorname{tr}\left(\mathbf{F}_{L}^{H}\left(\mathbf{H}_{L}^{H} \sum_{j=1}^{K} \mathbf{W}_{j}^{H} \mathbf{W}_{j} \mathbf{H}_{L}+\lambda_{L} \mathbf{I}_{N_{L-1}}\right) \mathbf{F}_{L}\right)}{P_{L-1}^{U L}}(34) \\
\lambda_{l} & =\frac{1}{P_{l}^{U L}} \operatorname{tr}\left(\mathbf { F } _ { l + 1 } ^ { H } \left(\bigotimes_{m=l+1}^{L-1} \mathbf{H}_{m}^{H} \mathbf{F}_{m+1}^{H} \mathbf{H}_{L}^{H} \sum_{j=1}^{K} \mathbf{W}_{j}^{H} \mathbf{W}_{j} \mathbf{H}_{L}\right.\right. \\
\times & \bigotimes_{m=L-1}^{l+1} \mathbf{F}_{m+1} \mathbf{H}_{m}+\sum_{k=l+2}^{L} \lambda_{k} \bigotimes_{m=l+1}^{k-1} \mathbf{H}_{m}^{H} \mathbf{F}_{m+1}^{H} \\
\left.\left.\times \bigotimes_{m=k-1}^{l+1} \mathbf{F}_{m+1} \mathbf{H}_{m}+\lambda_{l+1} \mathbf{I}_{N_{l}}\right) \mathbf{F}_{l+1}\right), l=1, \ldots, L-2 .(35) &
\end{aligned}
$$

Proof: See Appendix A in [10].

\section{B. The KKT Conditions of the Downlink Problem}

The Lagrangian function of (22)-(24) can be written as

$$
\begin{aligned}
\mathcal{L}^{D L} & =\sum_{j=1}^{K} \operatorname{tr}\left(\mathbf{E}_{j}^{D L}\right)+\alpha_{1}\left(\sum_{j=1}^{K} \operatorname{tr}\left(\mathbf{T}_{j} \mathbf{T}_{j}^{H}\right)-P_{1}^{D L}\right) \\
& +\sum_{l=2}^{L} \alpha_{l}\left(\operatorname{tr}\left(\mathbf{Z}_{l}\left(\mathbf{K}_{l-1} \mathbf{A}^{D L} \mathbf{K}_{l-1}^{H}+\mathbf{C}_{l-1}^{D L}\right) \mathbf{Z}_{l}^{H}\right)-P_{l}^{D L}\right)
\end{aligned}
$$

where $\alpha_{1}$ and $\alpha_{l}, l=2, \ldots, L$, are the Lagrange multipliers of the power constraints in (23) and (24). The gradient function of (36) with respect to $\mathbf{T}_{k}, \mathbf{Z}_{l}, \mathbf{D}_{k}$ is given by

$$
\begin{aligned}
& \mathbf{H}_{L} \mathbf{K}_{L-1}^{H} \mathbf{Z}_{L}^{H} \mathbf{G}_{k} \mathbf{D}_{k}^{H} \\
& =\left(\alpha_{1} \mathbf{I}_{M_{k}}+\sum_{l=2}^{L} \alpha_{l} \mathbf{H}_{L} \mathbf{K}_{l-1}^{H} \mathbf{Z}_{l}^{H} \mathbf{Z}_{l} \mathbf{K}_{l-1} \mathbf{H}_{L}^{H}\right. \\
& \left.+\sum_{j=1}^{K} \mathbf{H}_{L} \mathbf{K}_{L-1}^{H} \mathbf{Z}_{L}^{H} \mathbf{G}_{j} \mathbf{D}_{j}^{H} \mathbf{D}_{j} \mathbf{G}_{j}^{H} \mathbf{Z}_{L} \mathbf{K}_{L-1} \mathbf{H}_{L}^{H}\right) \mathbf{T}_{k},
\end{aligned}
$$

$$
\begin{aligned}
\sum_{j=1}^{K} \mathbf{X}_{k}^{(L)} \mathbf{G}_{j} \mathbf{D}_{j}^{H} \mathbf{T}_{j}^{H} \mathbf{H}_{L} \mathbf{Y}_{k}^{(1)} & \sum_{j=1}^{K} \mathbf{X}_{k}^{(L)} \mathbf{G}_{j} \mathbf{D}_{j}^{H} \mathbf{D}_{j} \mathbf{G}_{j}^{H} \mathbf{Z}_{L} \mathbf{K}_{L-1} \mathbf{A}^{D L} \mathbf{Y}_{k}^{(1)} \\
+ & \sum_{j=1}^{K} \mathbf{X}_{k}^{(L)} \mathbf{G}_{j} \mathbf{D}_{j} \mathbf{D}_{j}^{H} \mathbf{G}_{j}^{H} \mathbf{Z}_{L}\left(\sum_{c=1}^{k-1} \prod_{m=2}^{L-c}\left(\mathbf{H}_{m}^{H} \mathbf{Z}_{L-m+1}\right)\right. \\
& \left.\times \mathbf{Y}_{k}^{(c)}\right)+\alpha_{k} \mathbf{Z}_{k}\left(\mathbf{K}_{k-1} \mathbf{A}^{D L} \mathbf{Y}_{k}^{(1)}+\mathbf{C}_{k-1}^{D L}\right) \\
+ & \sum_{l=k+1}^{L} \alpha_{l} \mathbf{X}_{k}^{(l)} \mathbf{Z}_{l}\left(\mathbf{K}_{l-1} \mathbf{A}^{D L} \mathbf{Y}_{k}^{(1)}\right. \\
& \left.+\sum_{n=1}^{k-1} \bigotimes_{m=L-l+2}^{L-n} \mathbf{H}_{m}^{H} \mathbf{Z}_{L-m+1} \mathbf{Y}_{k}^{(n)}\right) \\
& \mathbf{T}_{k}^{H} \mathbf{H}_{L} \mathbf{K}_{L-1}^{H} \mathbf{Z}_{L}^{H} \mathbf{G}_{k} \\
& =\mathbf{D}_{k}\left(\mathbf{G}_{k}^{H} \mathbf{Z}_{L} \mathbf{K}_{L-1} \mathbf{A}^{D L} \mathbf{K}_{L-1}^{H} \mathbf{Z}_{L}^{H} \mathbf{G}_{k}+\mathbf{C}_{L}^{(k)}\right) .(39)
\end{aligned}
$$

The other KKT conditions associated with the problem (22)(24) for $l=2, \ldots, L$ are given below

$$
\begin{aligned}
& \alpha_{1}\left(\sum_{j=1}^{K} \operatorname{tr}\left(\mathbf{T}_{j} \mathbf{T}_{j}^{H}\right)-P_{1}^{D L}\right)=0 \\
& \alpha_{l}\left(\operatorname{tr}\left(\mathbf{Z}_{l}\left(\mathbf{Z}_{l-1} \mathbf{A}^{D L} \mathbf{Z}_{l-1}^{H}+\mathbf{C}_{l-1}^{D L}\right) \mathbf{Z}_{l}^{H}\right)-P_{l}^{D L}\right)=0 \\
& \alpha_{1} \geq 0, \quad \sum_{j=1}^{K} \operatorname{tr}\left(\mathbf{T}_{j} \mathbf{T}_{j}^{H}\right) \leq P_{1}^{D L} \\
& \alpha_{l} \geq 0, \quad \operatorname{tr}\left(\mathbf{Z}_{l}\left(\mathbf{Z}_{l-1} \mathbf{A}^{D L} \mathbf{Z}_{l-1}^{H}+\mathbf{C}_{l-1}^{D L}\right) \mathbf{Z}_{l}^{H}\right) \leq P_{l}^{D L} .
\end{aligned}
$$

In (38), $\mathbf{X}_{k}^{(c)}$ and $\mathbf{Y}_{k}^{(c)}$ are defined as

$$
\begin{aligned}
& \mathbf{X}_{k}^{(c)}= \begin{cases}\bigotimes_{m=L-k+1}^{L-c+2}\left(\mathbf{H}_{m} \mathbf{Z}_{L-m+2}^{H}\right), & \text { otherwise } \\
\mathbf{I}_{N_{1}}, & k=c\end{cases} \\
& \mathbf{Y}_{k}^{(c)}=\left\{\begin{array}{ll}
\bigotimes_{m=L-c}^{L-k+2}\left(\mathbf{Z}_{L-m+1}^{H} \mathbf{H}_{m}\right), & \text { otherwise } \\
\mathbf{I}_{N_{L-k+1}}, & k=c+1
\end{array} .\right.
\end{aligned}
$$

Lemma 2. For any solutions satisfying the KKT conditions (37)-(43), the Lagrange multipliers are

$$
\begin{aligned}
& \alpha_{L}=\frac{\sum_{k=1}^{K} \operatorname{tr}\left(\mathbf{D}_{k} \mathbf{D}_{k}^{H}\right)}{P_{L}^{D L}} \\
& \alpha_{L-1}=\frac{\operatorname{tr}\left(\mathbf{Z}_{L}^{H}\left(\sum_{i=1}^{K} \mathbf{G}_{i} \mathbf{D}_{i}^{H} \mathbf{D}_{i} \mathbf{G}_{i}^{H}+\alpha_{L} \mathbf{I}_{M_{i}}\right) \mathbf{Z}_{L}\right)}{P_{L-1}^{D L}} \\
& \alpha_{L-l+1}=\frac{1}{P_{L-l+1}^{D L}} \operatorname{tr}\left(\mathbf { Z } _ { L - l + 2 } ^ { H } \left(\bigotimes_{i=l-1}^{2} \mathbf{H}_{i} \mathbf{Z}_{L-i+2}^{H}\right.\right. \\
& \times \sum_{i=1}^{K} \mathbf{G}_{i} \mathbf{D}_{i}^{H} \mathbf{D}_{i} \mathbf{G}_{i}^{H} \bigotimes_{i=2}^{l-1} \mathbf{Z}_{L-i+2} \mathbf{H}_{i}^{H} \\
& +\sum_{j=2}^{l-1} \alpha_{L-j+2}\left[\bigotimes_{i=l-1}^{j} \mathbf{H}_{i} \mathbf{Z}_{L-i+2}^{H} \bigotimes_{i=j}^{l-1} \mathbf{Z}_{L-i+2} \mathbf{H}_{i}^{H}\right] \\
& \left.\left.+\alpha_{L-l+2} \mathbf{I}_{N_{l-1}}\right) \mathbf{Z}_{L-l+2}\right), \quad l=3, \ldots, L \text {. }
\end{aligned}
$$


Proof: Similar to the proof of Lemma 1, Lemma 2 can also be proved easily.

\section{Sum-MSE Uplink-Downlink Duality}

Theorem 1. Assume that the uplink transceiver matrices, $\left\{\mathbf{F}_{l}\right\}_{l=2}^{L},\left\{\mathbf{B}_{j}\right\}_{j=1}^{K},\left\{\mathbf{W}_{j}\right\}_{j=1}^{K}$ satisfy the uplink KKT conditions (26)-(32). Let $\mathbf{T}_{j}=\sqrt{1 / \lambda_{L}} \mathbf{W}_{j}^{H}, \mathbf{D}_{j}=\sqrt{\lambda_{1}} \mathbf{B}_{j}^{H}$, $\mathbf{Z}_{l}=\sqrt{\lambda_{L-l+2} / \lambda_{L-l+1}} \mathbf{F}_{L-l+2}^{H}, l=2, \ldots, L$. Then, when the power constraint of the $l$ th node of the downlink channel is swapped with the power constraint of the $(L-l+1)$ th node of the uplink channel, i.e., $P_{l}^{D L}=P_{L-l+1}^{U L}, l=$ $1, \ldots, L$, sum-MSE achieved by $\left\{\mathbf{F}_{l}\right\}_{l=2}^{L},\left\{\mathbf{B}_{j}\right\}_{j=1}^{K},\left\{\mathbf{W}_{j}\right\}_{j=1}^{K}$ can also be achieved by the downlink transceiver matrices, $\left\{\mathbf{Z}_{l}\right\}_{l=2}^{L},\left\{\mathbf{T}_{j}\right\}_{j=1}^{K},\left\{\mathbf{D}_{j}\right\}_{j=1}^{K}$, which satisfy the downlink KKT conditions (37)-(43). Conversely, assume that the downlink transceiver matrices $\left\{\mathbf{Z}_{l}\right\}_{l=2}^{L},\left\{\mathbf{T}_{j}\right\}_{j=1}^{K},\left\{\mathbf{D}_{j}\right\}_{j=1}^{K}$ satisfy the KKT conditions (37)-(43). Let $\mathbf{B}_{j}=\sqrt{1 / \alpha_{L}} \mathbf{D}_{j}^{H}, \mathbf{W}_{j}=$ $\sqrt{\alpha_{1}} \mathbf{T}_{j}^{H}$ and $\mathbf{F}_{L-l+2}=\sqrt{\alpha_{l} / \alpha_{l-1}} \mathbf{Z}_{l}^{H}, \quad l=2, \ldots, L$. Then, when the power constraint of the $l$ th node of the uplink channel is swapped with the power constraint of the $(L-l+1)$-th node of the downlink channel, i.e., $P_{l}^{U L}=P_{L-l+1}^{D L}, l=1, \ldots, L$, the sum-MSE achieved by $\left\{\mathbf{Z}_{l}\right\}_{l=2}^{L},\left\{\mathbf{T}_{j}\right\}_{j=1}^{K},\left\{\mathbf{D}_{j}\right\}_{j=1}^{K}$ can also be achieved by the uplink transceiver matrices $\left\{\mathbf{F}_{l}\right\}_{l=2}^{L},\left\{\mathbf{B}_{j}\right\}_{j=1}^{K},\left\{\mathbf{W}_{j}\right\}_{j=1}^{K}$, which satisfy the uplink KKT conditions (26)-(32).

Proof: See Appendix B in [10].

Theorem 1 shows that sum-MSE achieved by a transceiver design that satisfies the KKT conditions of an uplink optimization problem, can also be achieved by a transceiver design that satisfies the KKT conditions of a downlink optimization problem, and vice versa. Therefore, the downlink transceiver optimization problems can be solved through solving an equivalent uplink problem, and vice versa. Since the uplink and downlink optimization problems are non-convex, the KKT conditions are only necessary for local minimums in both channels. And by Theorem 1, every possible local minimum (satisfying the KKT conditions) of the uplink sum-MSE corresponds to a same local minimum in the downlink.

\section{Numerical EXAMPLES}

In this section, we simulate five-hop multiuser MIMO relay systems. For simplicity, we assume all users have the same number of antennas (i.e., $M_{i}=M, i=1, \cdots, K$ ) and all relay nodes and the destination node in the uplink have the same number of antennas (i.e., $N_{l}=N, l=$ $1, \cdots, L)$. We set $P_{L}^{U L}=P_{1}^{D L}=20 \mathrm{~dB}$ and assume that $P_{l}^{D L}=P_{L-l+1}^{U L}=P, \quad l=2, \cdots, L$. All simulation results are averaged over 1000 channel realizations. We use the iterative algorithm in [11] to design the optimal uplink transceivers $\left\{\mathbf{F}_{l}\right\}_{l=2}^{L},\left\{\mathbf{B}_{j}\right\}_{j=1}^{K},\left\{\mathbf{W}_{j}\right\}_{j=1}^{K}$ and use the proposed duality result to obtain the optimal downlink transceivers $\left\{\mathbf{Z}_{l}\right\}_{l=2}^{L},\left\{\mathbf{T}_{j}\right\}_{j=1}^{K},\left\{\mathbf{D}_{j}\right\}_{j=1}^{K}$. Fig. 3 shows the MSE performance of the uplink and downlink systems versus $P$ with $K=3, M=2$, and $N=10$. It can be seen from

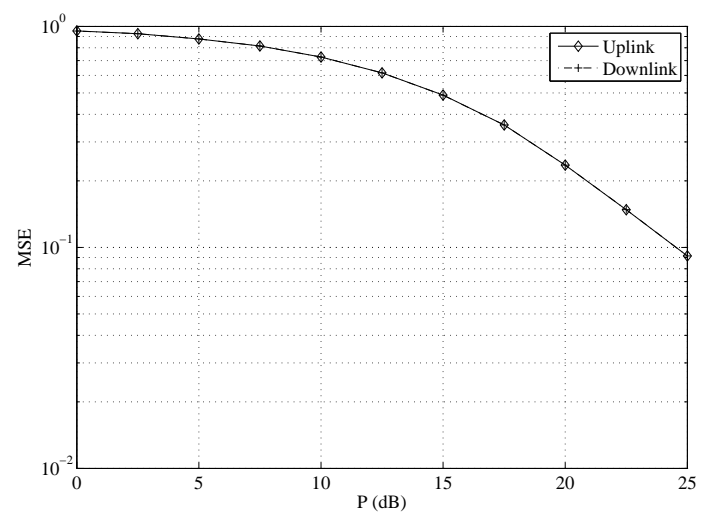

Fig. 3. MSE versus $P$. $K=3, M=2, N=10, P_{L}^{U L}=P_{1}^{D L}=20 \mathrm{~dB}$, $P_{l}^{D L}=P_{L-l+1}^{U L}=P, l=2, \cdots, L$.

Figs. 3 that the curves overlap, indicating that both the uplink and downlink systems achieve the same sum-MSE.

\section{CONCLUSION}

We have established the uplink-downlink sum-MSE duality in a multi-hop AF MIMO relay system, which is a generalization of several sum-MSE duality results. By analyzing the KKT conditions of the uplink and downlink minimum sumMSE transceiver optimization problems, it is shown that both the uplink and the downlink systems share the same achievable sum-MSE region.

\section{REFERENCES}

[1] S. Shi and M. Schubert, "MMSE transmit optimization for multi-user multi-antenna systems," in Proc. IEEE Int. Conf. Acoustics, Speech, and Signal Process. (ICASSP), Philadelphia, PA, Mar. 2005.

[2] A. Khachan, A. J. Tenenbaum, and R. Adve, "Linear processing for the downlink in multiuser MIMO systems with multiple data streams," in Proc. IEEE Int. Conf. Commun., vol. 9, pp. 4113-4118, June 2006.

[3] M. Ding and S. D. Blostein, "Uplink-downlink duality in normalized MSE or SINR under imperfect channel knowledge," in Proc. IEEE GLOBECOM 07, pp. 3786-3790, Nov. 2007.

[4] M. B. Shenouda and T. Davidson, "On the design of linear transceivers for multiuser systems with channel uncertainty," IEEE J. Sel. Areas Commun., vol. 26, no. 6, pp. 1015-1024, Aug. 2008

[5] M. Ding and S. D. Blostein, "Relation between joint optimizations for multiuser MIMO uplink and downlink with imperfect CSI," IEEE ICASSP, pp. 3149-3152, Apr. 2008.

[6] J. Liu and Z. Qiu, "Sum MSE uplink-downlink duality of multiuser amplify-and-forward MIMO relay systems," in Proc. IEEE Vehicular Techn. Conf. (VTC Fall), pp.1-5, Sept. 2011.

[7] A. C. Cirik, Y. Rong, Y. Ma, and Y. Hua, "On MAC-BC duality of multihop MIMO relay channel with imperfect channel knowledge," to appear in IEEE Trans. Wireless Commun, April 2014.

[8] Y. Rong and M. R. A. Khandaker, "On uplink-downlink duality of multihop MIMO relay channel," IEEE Trans. Wireless Commun., vol. 10, pp. 1923-1931, Jun. 2011.

[9] A. Hjorungnes and D. Gesbert, "Complex-valued matrix differentiations: Techniques and key results," IEEE Trans. Signal Process., vol. 55, no. 6, pp. 2740-2746, Jun. 2007.

[10] A. C. Cirik, Muhammad R. A. Khandaker, Y. Rong and Y. Hua, "On uplink-downlink sum-MSE duality of multi-hop MIMO relay channel," unpublished.

[11] M. R. A. Khandaker and Y. Rong, "Joint transceiver optimization for multiuser MIMO relay communication systems," IEEE Trans. Signal Process., vol. 60, pp. 5977-5986, Nov. 2012. 